\title{
Identification of RUNX2 variants associated with cleidocranial dysplasia
}

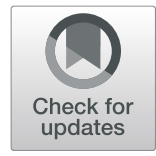

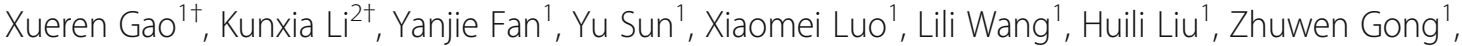 \\ Jianguo Wang ${ }^{1}$, Yu Wang ${ }^{1}$, Xuefan $\mathrm{Gu}^{1^{*}}$ and Yongguo $\mathrm{Yu}^{{ }^{*}}$ (D)
}

\begin{abstract}
Background: Cleidocranial dysplasia (CCD) is a rare autosomal dominant disorder mainly characterized by hypoplastic or absent clavicles, delayed closure of the fontanelles, multiple dental abnormalities, and short stature. Runt-related transcription factor 2 (RUNX2) gene variants can cause CCD, but are not identified in all CCD patients.

Methods: In this study, we detected genetic variants in seven unrelated children with CCD by targeted highthroughput DNA sequencing or Sanger sequencing.

Results: All patients carried a RUNX2 variant, totally including three novel pathogenic variants (c.722_725delTGT, p.Leu241Serfs*8; c.231_232delTG, Ala78Glyfs*82; c.909C > G, p.Tyr303*), three reported pathogenic variants (c.577C > T, p.Arg193*; c.574G > A, p.Gly192Arg; c.673 C > T, p.Arg225Trp), one likely pathogenic variant (c.668G > T, p.Gly223Val). The analysis of the variant source showed that all variants were de novo except the two variants (c.909C > G, p.Tyr303*; c.668G > T, p.Gly223Val) inherited from the patient's father and mother with CCD respectively. Further bioinformatics analysis indicated that these variants could influence the structure of RUNX2 protein by changing the number of $\mathrm{H}$-bonds or amino acids. The experimental result showed that the Gly223Val mutation made RUNX2 protein unable to quantitatively accumulate in the nucleus.
\end{abstract}

Conclusions: The present study expands the pathogenic variant spectrum of RUNX2 gene, which will contribute to the diagnosis of CCD and better genetic counseling in the future.

Keywords: RUNX2, Pathogenic variant, Cleidocranial dysplasia, Targeted next-generation sequencing

\section{Background}

Cleidocranial dysplasia (CCD; OMIM \#119600) is a rare autosomal dominant disorder mainly characterised by hypoplastic or absent clavicles, delayed closure of fontanelles, multiple dental abnormalities, and short stature [1-3]. Variants in runt-related transcription factor $2(R U N X 2)$ gene $(\mathrm{OMIM} * 600211)$ can result in haploinsufficiency of the protein and have been related to CCD $[1,2]$. The RUNX2 gene is located on chromosome $6 \mathrm{p} 21.1$ and encodes a transcription factor with a highly conserved Runt domain $[4,5]$. The Runt domain is responsible for binding to a specific

\footnotetext{
* Correspondence: guxuefan@xinhuamed.com.cn; yuyongguo@shsmu.edu.cn ${ }^{+}$Xueren Gao and Kunxia Li contributed equally to this work.

${ }^{1}$ Department of Pediatric Endocrinology and Genetics, Shanghai Institute for Pediatric Research, Xinhua Hospital, School of Medicine, Shanghai Jiaotong University, Shanghai 200092, China

Full list of author information is available at the end of the article
}

DNA motif $\left(\mathrm{TG}^{\mathrm{T}} / \mathrm{C} \mathrm{GGT}\right.$ sequence) in the promoter region of its target genes and heterodimerization with CBFB (core-binding factor subunit beta) [6-8]. The former participates in regulating the transcription of multiple genes. The latter increases the DNA-binding affinity as well as protects and stabilizes RUNX2 against proteolytic degradation. The $\mathrm{N}$-terminal side of the Runt domain links a Q/A region consisting of 23 consecutive glutamine residues followed by 17 alanine residues, which acts as a second transactivation domain [9]. The C-terminal side of the Runt domain links a PST (proline/serine/threonine)-rich region, which contains the phosphorylation sites and represents the third transactivation domain $[9,10]$. The last five amino acids (VWRPY) of RUNX2 protein compose a conserved motif in all runt proteins, and functions as a transcriptional repression domain $[9,11]$.

(C) The Author(s). 2019 Open Access This article is distributed under the terms of the Creative Commons Attribution 4.0 International License (http://creativecommons.org/licenses/by/4.0/), which permits unrestricted use, distribution, and 
RUNX2 is essential for osteoblastic differentiation and skeletal morphogenesis. In mouse models, the homozygous mutation of RUNX2 gene blocked both intramembranous and endochondral ossification and resulted in a complete lack of bone formation [12]. The heterozygous mutation $\left(R U N X 2^{+/-}\right)$caused a similar phenotype to that of human CCD [13]. To date, 184 publicly available mutations in RUNX2 gene have been deposited in the Human Gene Mutation Database (HGMD, www.hgmd.cf.ac.uk). Most of these mutations were missense and clustered in Runt domain. Additionally, nonsense mutations, insertions or deletions are also observed in the RUNX2 gene, which are predominant within the $\mathrm{Q} /$ A domain or the PST domain. Although many mutations in the RUNX2 gene have been identified in familial and sporadic cases, novel mutation is still reported recently, suggesting that mutational screening on RUNX2 gene is far from saturation [14-19].

In the present study, we conducted genetic evaluation for a cohort of seven Chinese children with CCD by targeted high-throughput DNA sequencing or Sanger sequencing, and found seven different variants in RUNX2 gene, including six pathogenic variants and one likely pathogenic variant. These results will contribute to the diagnosis of $\mathrm{CCD}$ and better genetic counseling in the future.

\section{Material and methods}

\section{Genomic DNA extraction and genetic testing}

A total of seven unrelated children with CCD ranging in age from 1 month to 12 years were enrolled for genetic evaluation (Table 1). Genomic DNA of probands and their family members was extracted from peripheral blood leukocytes using Lab-Aid Nucleic Acid Isolation Kit (Zeesan, China), according to the manufacturer's instructions.
Among these $\mathrm{CCD}$ patients, five patients were firstly detected by targeted high-throughput DNA sequencing, two patients directly by Sanger sequencing (Table 1). For targeted high-throughput DNA sequencing, the preparation of sequencing library was completed using Agilent Inherited Disease panel, Agilent Focused exome panel or xGen Exome research panel v1.0 (Integrated DNA Technologies, Coralville, Iowa). Sequencing was performed on the Illumina HiSeq 2500 or 4000 (Illumina, San Diego, $\mathrm{CA})$, according to the manufacturer's instructions. Burrows-Wheeler Aligner (BWA, version 0.7.10) was used to mapping reads to the human reference genome (GRCh37/hg19). Base calling, QC analysis and coverage analysis were performed with Picard tools1.124 and GATK software. Variants were annotated using SnpEff version 4.2. Subsequently, the following variants were filtered out: (i) variants with $>1 \%$ frequency in the population variant databases including 1000 Genomes Project, Exome Variant Server (EVS) and Exome Aggregation Consortium (ExAC) or $>5 \%$ frequency in our inhouse database (based on 150 exome datasets), (ii) intergenic and $3^{\prime} / 5^{\prime}$ untranslated region variants, none splice-related intronic and synonymous variants.

For Sanger sequencing, all exons of the RUNX2 gene in these probands were amplified by PCR reaction. DNA sequence variants were identified by Mutation Surveyor V4.0.5 software with reference sequences (NG_008020.1).

\section{Variant assessment}

MutationTaster (http://www.mutationtaster.org), SIFT (http://sift.jcvi.org), and PolyPhen-2 (http://genetics. bwh.harvard.edu/pph2/) were used to assess pathogenic potential of the variants [20-22]. Combined with clinical manifestation and modes of inheritance, candidate variants were validated by Sanger sequencing for all

Table 1 Genetic detection methods and basic characteristics of seven children with CCD

\begin{tabular}{|c|c|c|c|c|}
\hline Proband ID & Gender & Age & Family history & Genetic detection methods \\
\hline Family_A_II1 & Male & $3 Y$ & No & $\begin{array}{l}\text { Inherited disease panel (Agilent) Hiseq4000(Illumina), } \\
\text { Sanger sequencing }\end{array}$ \\
\hline Family_B_II1 & Female & $1 Y 9 M$ & No & $\begin{array}{l}\text { Focused exome panel (Agilent) Hiseq2500(Illumina), } \\
\text { Sanger sequencing }\end{array}$ \\
\hline Family_C_II1 & Male & $9 Y 11 \mathrm{M}$ & No & Sanger sequencing \\
\hline Family_D_II1 & Male & $12 Y$ & No & $\begin{array}{l}\text { xGen Exome research panel v1.0 (IDT) HiSeq4000(Illumina), } \\
\text { Sanger sequencing }\end{array}$ \\
\hline Family_E_II1 & Female & $1 \mathrm{M}$ & No & Sanger sequencing \\
\hline Family_F_III1 & Male & $3 Y$ & Father with CCD & $\begin{array}{l}\text { xGen Exome research panel v1.0 (IDT) HiSeq4000(Illumina), } \\
\text { Sanger sequencing }\end{array}$ \\
\hline Family_G_III1 & Male & $6 Y$ & $\begin{array}{l}\text { Mother with CCD Uncle with CCD } \\
\text { Grandmother with CCD }\end{array}$ & $\begin{array}{l}\text { xGen Exome research panel v1.0 (IDT) HiSeq4000(Illumina), } \\
\text { Sanger sequencing }\end{array}$ \\
\hline
\end{tabular}


Table 2 Comparison of clinical features of CCD children with different RUNX2 gene variant

\begin{tabular}{|c|c|c|c|c|c|c|c|}
\hline Clinical synopsis & $\begin{array}{l}\text { Family_A_111 } \\
(c .577 \mathrm{C}>\mathrm{T})\end{array}$ & $\begin{array}{l}\text { Family_B_II1 } \\
(\text { c.574G > A) }\end{array}$ & $\begin{array}{l}\text { Family_C_II1 } \\
(c .673 C>T)\end{array}$ & $\begin{array}{l}\text { Family_D_II1 } \\
\text { (c.722_725delTGTT) }\end{array}$ & $\begin{array}{l}\text { Family_E_II1 } \\
\text { (c.231_232delTG) }\end{array}$ & $\begin{array}{l}\text { Family_F_III1 } \\
(\text { c. } 909 \mathrm{C}>\mathrm{G})\end{array}$ & $\begin{array}{l}\text { Family_G_III1 } \\
(c .668 G>T)\end{array}$ \\
\hline \multicolumn{8}{|l|}{ GROWTH } \\
\hline \multicolumn{8}{|l|}{ Height } \\
\hline Short stature & $\sqrt{ }$ & $\sqrt{ }$ & $\sqrt{ }$ & $\sqrt{ }$ & $\sqrt{ }$ & $\sqrt{ }$ & $\sqrt{ }$ \\
\hline \multicolumn{8}{|l|}{ HEAD \& NECK } \\
\hline \multicolumn{8}{|l|}{ Head } \\
\hline Delayed fontanelle closure & $\sqrt{ }$ & $\sqrt{ }$ & $\sqrt{ }$ & $\sqrt{ }$ & $\sqrt{ }$ & $\sqrt{ }$ & $\sqrt{ }$ \\
\hline Parietal bossing & $\sqrt{ }$ & $\sqrt{ }$ & $\sqrt{ }$ & $\sqrt{ }$ & $\sqrt{ }$ & $\sqrt{ }$ & $\sqrt{ }$ \\
\hline \multicolumn{8}{|l|}{ Anterior fontanelle open in adults } \\
\hline \multicolumn{8}{|l|}{ Face } \\
\hline Frontal bossing & $\sqrt{ }$ & $\sqrt{ }$ & $\sqrt{ }$ & $\sqrt{ }$ & $\sqrt{ }$ & $\sqrt{ }$ & $\sqrt{ }$ \\
\hline Metopic groove & $\sqrt{ }$ & $\sqrt{ }$ & $\sqrt{ }$ & $\sqrt{ }$ & $\sqrt{ }$ & $\sqrt{ }$ & $\sqrt{ }$ \\
\hline Midface hypoplasia & $\sqrt{ }$ & $\sqrt{ }$ & $\sqrt{ }$ & $\sqrt{ }$ & $\sqrt{ }$ & $\sqrt{ }$ & $\sqrt{ }$ \\
\hline Micrognathia & $\sqrt{ }$ & $\sqrt{ }$ & $\sqrt{ }$ & $\sqrt{ }$ & $\sqrt{ }$ & $\sqrt{ }$ & $\sqrt{ }$ \\
\hline \multicolumn{8}{|l|}{ Ears } \\
\hline \multicolumn{8}{|l|}{ Deafness } \\
\hline \multicolumn{8}{|l|}{ Eyes } \\
\hline Hypertelorism & & $\sqrt{ }$ & $\sqrt{ }$ & $\sqrt{ }$ & $\sqrt{ }$ & $\sqrt{ }$ & $\sqrt{ }$ \\
\hline \multicolumn{8}{|l|}{ Nose } \\
\hline Low nasal bridge & $\sqrt{ }$ & $\sqrt{ }$ & $\sqrt{ }$ & $\sqrt{ }$ & $\sqrt{ }$ & $\sqrt{ }$ & $\sqrt{ }$ \\
\hline \multicolumn{8}{|l|}{ Mouth } \\
\hline \multicolumn{8}{|l|}{ Cleft palate } \\
\hline \multicolumn{8}{|l|}{ Narrow, high-arched palate } \\
\hline \multicolumn{8}{|l|}{ Teeth } \\
\hline $\begin{array}{l}\text { Delayed eruption of } \\
\text { deciduous teeth }\end{array}$ & $\sqrt{ }$ & $\sqrt{ }$ & $\sqrt{ }$ & $\sqrt{ }$ & $\sqrt{ }$ & $\sqrt{ }$ & $\sqrt{ }$ \\
\hline \multicolumn{8}{|c|}{ Delayed eruption of permanent teeth } \\
\hline Supernumerary teeth & & & $\sqrt{ }$ & $\sqrt{ }$ & $\sqrt{ }$ & $\sqrt{ }$ & $\sqrt{ }$ \\
\hline Retention cysts & & & $\sqrt{ }$ & $\sqrt{ }$ & $\sqrt{ }$ & $\sqrt{ }$ & $\sqrt{ }$ \\
\hline Enamel hypoplasia & $\sqrt{ }$ & $\sqrt{ }$ & $\sqrt{ }$ & $\sqrt{ }$ & $\sqrt{ }$ & $\sqrt{ }$ & $\sqrt{ }$ \\
\hline \multicolumn{8}{|l|}{ RESPIRATORY } \\
\hline \multicolumn{8}{|l|}{ Airways } \\
\hline \multicolumn{8}{|l|}{$\begin{array}{l}\text { Respiratory distress in } \\
\text { early infancy }\end{array}$} \\
\hline \multicolumn{8}{|l|}{ CHEST } \\
\hline \multicolumn{8}{|l|}{ External Features } \\
\hline Narrow thorax & $\sqrt{ }$ & $\sqrt{ }$ & $\sqrt{ }$ & $\sqrt{ }$ & $\sqrt{ }$ & $\sqrt{ }$ & $\sqrt{ }$ \\
\hline $\begin{array}{l}\text { Abnormal facility in opposing } \\
\text { the shoulders }\end{array}$ & $\sqrt{ }$ & $\sqrt{ }$ & $\sqrt{ }$ & $\sqrt{ }$ & $\sqrt{ }$ & $\sqrt{ }$ & $\sqrt{ }$ \\
\hline \multicolumn{8}{|l|}{ Ribs Sternum Clavicles \& Scapulae } \\
\hline \multicolumn{8}{|l|}{ Small scapula } \\
\hline Hypoplastic clavicles & $\sqrt{ }$ & $\sqrt{ }$ & $\sqrt{ }$ & $\sqrt{ }$ & $\sqrt{ }$ & $\sqrt{ }$ & $\sqrt{ }$ \\
\hline \multicolumn{8}{|l|}{ Aplastic clavicles } \\
\hline \multicolumn{8}{|l|}{ Short ribs } \\
\hline Cervical ribs & & & & & & & \\
\hline
\end{tabular}


Table 2 Comparison of clinical features of CCD children with different RUNX2 gene variant (Continued)

\begin{tabular}{|c|c|c|c|c|c|c|c|}
\hline Clinical synopsis & $\begin{array}{l}\text { Family_A_II1 } \\
(c .577 \mathrm{C}>\mathrm{T})\end{array}$ & $\begin{array}{l}\text { Family_B_II1 } \\
(\text { c.574G }>\text { A) }\end{array}$ & $\begin{array}{l}\text { Family_C_II1 } \\
(c .673 C>T)\end{array}$ & $\begin{array}{l}\text { Family_D_II1 } \\
\text { (c.722_725delTGTT) }\end{array}$ & $\begin{array}{l}\text { Family_E_II1 } \\
\text { (c.231_232delTG) }\end{array}$ & $\begin{array}{l}\text { Family_F_III1 } \\
(c .909 C>\text { G) }\end{array}$ & $\begin{array}{l}\text { Family_G_III1 } \\
(c .668 G>T)\end{array}$ \\
\hline \multicolumn{8}{|l|}{ SKELETAL } \\
\hline \multicolumn{8}{|l|}{ Osteosclerosis } \\
\hline Increased bone fragility & $\sqrt{ }$ & $\sqrt{ }$ & $\sqrt{ }$ & $\sqrt{ }$ & $\sqrt{ }$ & $\sqrt{ }$ & $\sqrt{ }$ \\
\hline \multicolumn{8}{|l|}{ Skull } \\
\hline Wormian bones & $\sqrt{ }$ & $\sqrt{ }$ & $\sqrt{ }$ & $\sqrt{ }$ & $\sqrt{ }$ & $\sqrt{ }$ & $\sqrt{ }$ \\
\hline Bossing of frontal bone & $\sqrt{ }$ & $\sqrt{ }$ & $\sqrt{ }$ & $\sqrt{ }$ & $\sqrt{ }$ & $\sqrt{ }$ & $\sqrt{ }$ \\
\hline \multicolumn{8}{|l|}{ Bossing of occipital bone } \\
\hline Bossing of parietal bone & $\sqrt{ }$ & $\sqrt{ }$ & $\sqrt{ }$ & $\sqrt{ }$ & $\sqrt{ }$ & $\sqrt{ }$ & $\sqrt{ }$ \\
\hline \multicolumn{8}{|l|}{ Calvarial thickening } \\
\hline \multicolumn{8}{|l|}{ Absent frontal sinuses } \\
\hline \multicolumn{8}{|l|}{ Absent paranasal sinuses } \\
\hline \multicolumn{8}{|l|}{ Hypoplastic frontal sinuses } \\
\hline \multicolumn{8}{|l|}{ Hypoplastic paranasal sinuses } \\
\hline \multicolumn{8}{|l|}{ Large foramen magnum } \\
\hline \multicolumn{8}{|l|}{ Spine } \\
\hline \multicolumn{8}{|l|}{ Spondylolysis } \\
\hline \multicolumn{8}{|l|}{ Spondylolisthesis } \\
\hline Scoliosis & $\sqrt{ }$ & $\sqrt{ }$ & $\sqrt{ }$ & $\sqrt{ }$ & $\sqrt{ }$ & $\sqrt{ }$ & $\sqrt{ }$ \\
\hline \multicolumn{8}{|l|}{ Kyphosis } \\
\hline \multicolumn{8}{|l|}{ Pelvis } \\
\hline \multicolumn{8}{|l|}{ Wide pubic symphysis } \\
\hline $\begin{array}{l}\text { Delayed mineralization of } \\
\text { pubic bone }\end{array}$ & $\sqrt{ }$ & $\sqrt{ }$ & $\sqrt{ }$ & $\sqrt{ }$ & $\sqrt{ }$ & $\sqrt{ }$ & $\sqrt{ }$ \\
\hline $\begin{array}{l}\text { Broad femoral head with } \\
\text { short femoral neck }\end{array}$ & $\sqrt{ }$ & $\sqrt{ }$ & $\sqrt{ }$ & $\sqrt{ }$ & $\sqrt{ }$ & $\sqrt{ }$ & $\sqrt{ }$ \\
\hline \multicolumn{8}{|l|}{ Coxa vara } \\
\hline Hypoplastic iliac wing & $\sqrt{ }$ & $\sqrt{ }$ & $\sqrt{ }$ & $\sqrt{ }$ & $\sqrt{ }$ & $\sqrt{ }$ & $\sqrt{ }$ \\
\hline \multicolumn{8}{|l|}{ Hands } \\
\hline \multicolumn{8}{|l|}{ Brachydactyly } \\
\hline Long second metacarpal & & & $\sqrt{ }$ & $\sqrt{ }$ & $\sqrt{ }$ & $\sqrt{ }$ & $\sqrt{ }$ \\
\hline \multicolumn{8}{|l|}{$\begin{array}{l}\text { Short middle phalanges of } \\
\text { second and fifth fingers }\end{array}$} \\
\hline \multicolumn{8}{|l|}{$\begin{array}{l}\text { Cone-shaped phalangeal } \\
\text { epiphyses }\end{array}$} \\
\hline \multicolumn{8}{|l|}{ NEUROLOGIC } \\
\hline \multicolumn{8}{|l|}{ Peripheral Nervous System } \\
\hline Syringomyelia & $\sqrt{ }$ & $\sqrt{ }$ & $\sqrt{ }$ & $\sqrt{ }$ & $\sqrt{ }$ & $\sqrt{ }$ & $\sqrt{ }$ \\
\hline
\end{tabular}

family members, and classified according to standards and guidelines of the American College of Medical Genetics and Genomics (ACMG). For the putative pathogenic or likely pathogenic variants, SWISSMODEL (https://swissmodel.expasy.org) and SwissPdbViewer 4.1 software (http://spdbv.vital-it.ch/) were used to analyze the effect of these variants on protein structure [23, 24].

\section{Subcellular localization of the RUNX2 mutant protein}

The cDNA of wide-type RUNX2 gene was synthesized by Sangon Biotech (Shanghai) Co., Ltd., and amplified by PCR. The forward primer was 5'-GACACAGATC TCGAGATGGCATCAAACAGCCTCTTCAGC-3' and the reverse primer was $5^{\prime}$-GTGTCGTCGACTGATATG GTCGCCAAACAGATTCA-3'. The PCR fragment was subcloned into pEGFP-N1 vector with the XhoI and SaII 
restriction sites. The RUNX2 668G $>\mathrm{T}$ (Gly223Val) mutation was introduced into pEGFP-N1 vector with wide-type RUNX2 cDNA by site-directed mutagenesis. The mutant primers were 5'-GCCTTCTGGGTTCC CGAGGTACATCTACTGTAACTTT AAT-3', and 5'ATTAAAGTTACAGTAGATGTACCTCGGGAA

CCCAGAAGGC-3'. All recombinant vectors were fully sequenced to exclude any additional mutations. The empty vector acting as a negative control (NC) and pEGFP-N1 vectors bearing wild type (WT) and mutant (Mut) were transfected into U2OS cells by using lipofectamine 2000 (Invitrogen). The cells were visualized and photographed (magnification 10X and 40X) with a fluorescent microscope (Olympus IX73, Japan).

\section{Results}

\section{Clinical features of CCD children}

All children underwent a clinical evaluation and were diagnosed as CCD by an experienced pediatrician. The clinical features of these patients including two female and five male patients were summarized in Table 2 . Besides the clavicle and skull dysplasia, short stature, scoliosis, enamel hypoplasia, delayed eruption of deciduous teeth, low nasal bridge, delayed mineralization

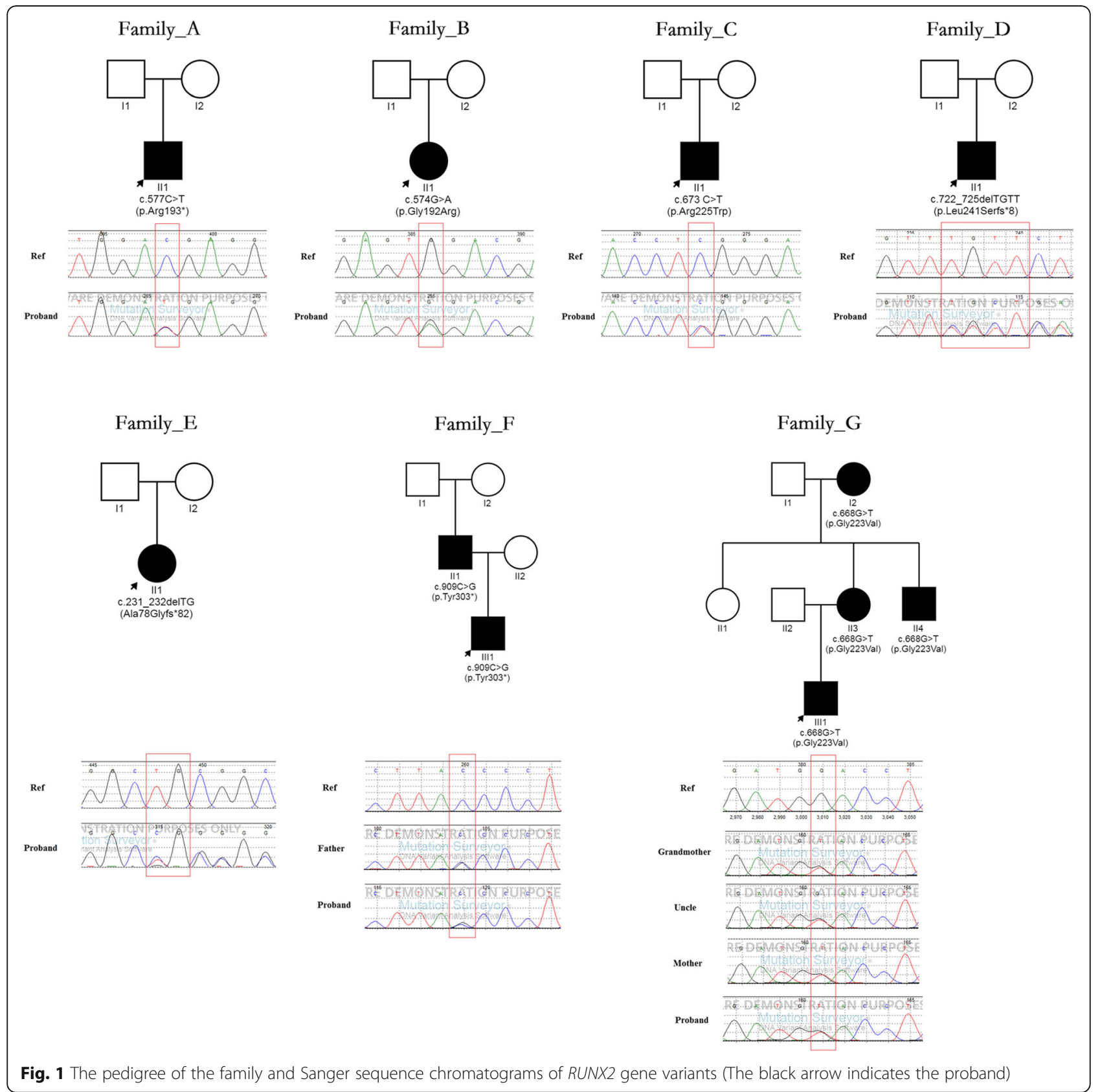




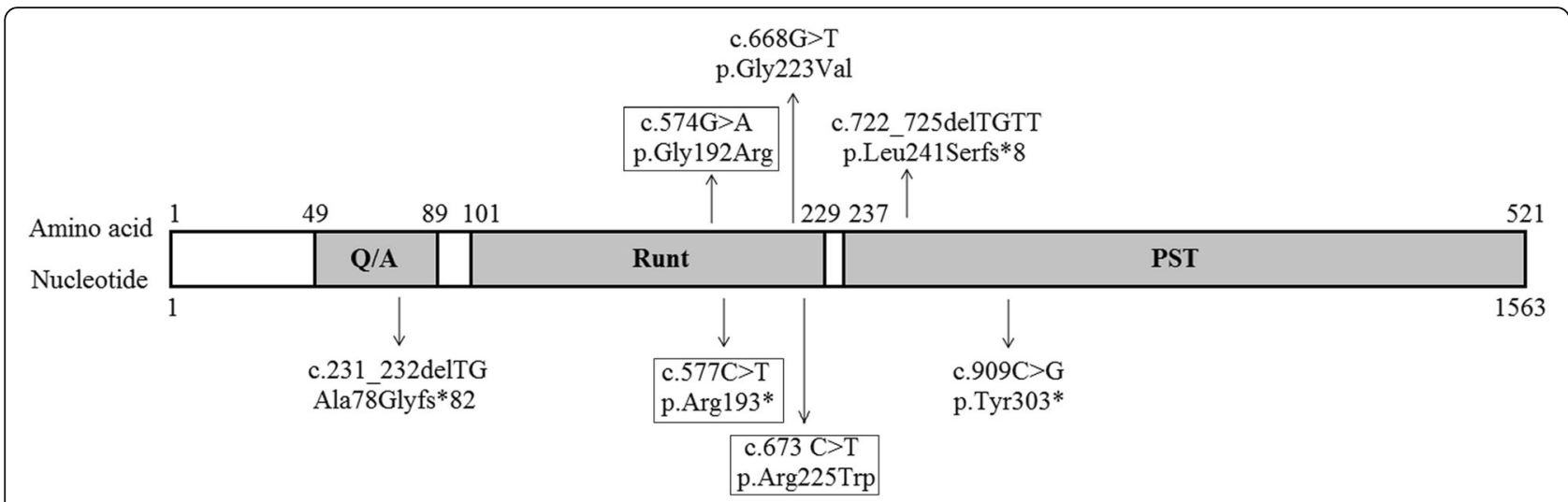

Fig. 2 Relative positions of RUNX2 gene variants identified in seven children with CCD (Variants in the box indicates the reported variants)

of pubic bone, broad femoral head with short femoral neck, hypoplastic iliac wing, syringomyelia and special faces were also observed in CCD children. Furthermore, hypertelorism was observed in all CCD children, except Family_A_II1. Supernumerary teeth, retention cysts and long second metacarpal were observed in all CCD children, except Family_A_II1 and Family_B_II1.

\section{Genetic testing}

All patients carried a $R U N X 2$ variant, totally including four novel variants and three reported variants (Figs. 1, 2 and Table 3). Among the seven variants, there were two pathogenic missense variants (c.574G > A, p.Gly192Arg; c.673 C > T, p.Arg225Trp), one likely pathogenic missense variant (c.668G > T, p.Gly223Val), two pathogenic frameshift variants (c.722_725delTGTT, p.Leu241Serfs*8; c.231_ 232delTG, Ala78Glyfs*82), and two pathogenic stop-gain variants (c.577C > T, p.Arg193*; c.909C > G, p.Tyr303*). The analysis of the variant source showed that all variants were de novo except the two variants (c.909C $>\mathrm{G}$, p.Tyr303*; c.668G > T, p.Gly223Val). The former variant was inherited from the patient's father with $C C D$, who carried a de novo heterozygous RUNX2 variant (c.909C > G, p.Tyr303*). The latter variant was inherited from the patient's mother with CCD, who carried a maternal inherited and heterozygous RUNX2 variant (c.668G > T, p.Gly223Val).

Table 3 Summarization of RUNX2 gene variants in seven children with CCD

\begin{tabular}{|c|c|c|c|c|c|c|c|c|}
\hline \multirow[t]{2}{*}{ Proband ID } & \multirow[t]{2}{*}{ Variant location } & \multirow[t]{2}{*}{ Variant type } & \multirow{2}{*}{$\begin{array}{l}\text { Variant } \\
\text { source }\end{array}$} & \multirow[t]{2}{*}{ Literature report } & \multicolumn{3}{|c|}{ Bioinformatic prediction } & \multirow{2}{*}{$\begin{array}{l}\text { ACMG } \\
\text { classification }\end{array}$} \\
\hline & & & & & MutationTaster & SIFT & PolyPhen-2 & \\
\hline Family_A_II1 & $\begin{array}{l}\text { NM_001024630.3: } \\
\text { c.577C > T, } \\
\text { p.Arg193* (Het) }\end{array}$ & Stopgain & De novo & $\begin{array}{l}\text { Hum Mol Genet. } \\
\text { 1999;8 (12):2311-6. }\end{array}$ & $\begin{array}{l}\text { Disease } \\
\text { causing }\end{array}$ & NA & NA & Pathogenic \\
\hline Family_B_II1 & $\begin{array}{l}\text { NM_001024630.3: } \\
\text { c.574G > A, } \\
\text { p.Gly192Arg (Het) }\end{array}$ & Missense & De novo & $\begin{array}{l}\text { J Hum Genet. } \\
\text { 2005;50 (12):679-83. }\end{array}$ & $\begin{array}{l}\text { Disease } \\
\text { causing }\end{array}$ & Damaging & $\begin{array}{l}\text { Probably } \\
\text { damaging }\end{array}$ & Pathogenic \\
\hline Family_C_II1 & $\begin{array}{l}\text { NM_001024630.3: } \\
\text { c.673 C > T, } \\
\text { p.Arg225Trp (Het) }\end{array}$ & Missense & De novo & $\begin{array}{l}\text { Am J Hum Genet. } \\
\text { 1999;65 (5):1268-78. }\end{array}$ & $\begin{array}{l}\text { Disease } \\
\text { causing }\end{array}$ & Damaging & $\begin{array}{l}\text { Probably } \\
\text { damaging }\end{array}$ & Pathogenic \\
\hline Family_D_II1 & $\begin{array}{l}\text { NM_001024630.3: } \\
\text { c.722_725delTGTT, } \\
\text { p.Leu241Serfs*8 (Het) }\end{array}$ & Frameshift & De novo & - & $\begin{array}{l}\text { Disease } \\
\text { causing }\end{array}$ & NA & NA & Pathogenic \\
\hline Family_E_II1 & $\begin{array}{l}\text { NM_001024630.3: } \\
\text { c.231_232delTG, } \\
\text { Ala78Glyfs*82 (Het) }\end{array}$ & Frameshift & De novo & - & $\begin{array}{l}\text { Disease } \\
\text { causing }\end{array}$ & NA & NA & Pathogenic \\
\hline Family_F_III1 & $\begin{array}{l}\text { NM_001024630.3: } \\
\text { c.909C > G, } \\
\text { p.Tyr303* (Het) }\end{array}$ & Stopgain & $\begin{array}{l}\text { Paternal } \\
\text { inheritance }\end{array}$ & - & $\begin{array}{l}\text { Disease } \\
\text { causing }\end{array}$ & NA & NA & Pathogenic \\
\hline Family_G_III1 & $\begin{array}{l}\text { NM_001024630.3: } \\
\text { c.668G > T, } \\
\text { p.Gly223Val (Het) }\end{array}$ & Missense & $\begin{array}{l}\text { Maternal } \\
\text { inheritance }\end{array}$ & - & $\begin{array}{l}\text { Disease } \\
\text { causing }\end{array}$ & Damaging & $\begin{array}{l}\text { Probably } \\
\text { damaging }\end{array}$ & $\begin{array}{l}\text { Likely } \\
\text { pathogenic }\end{array}$ \\
\hline
\end{tabular}




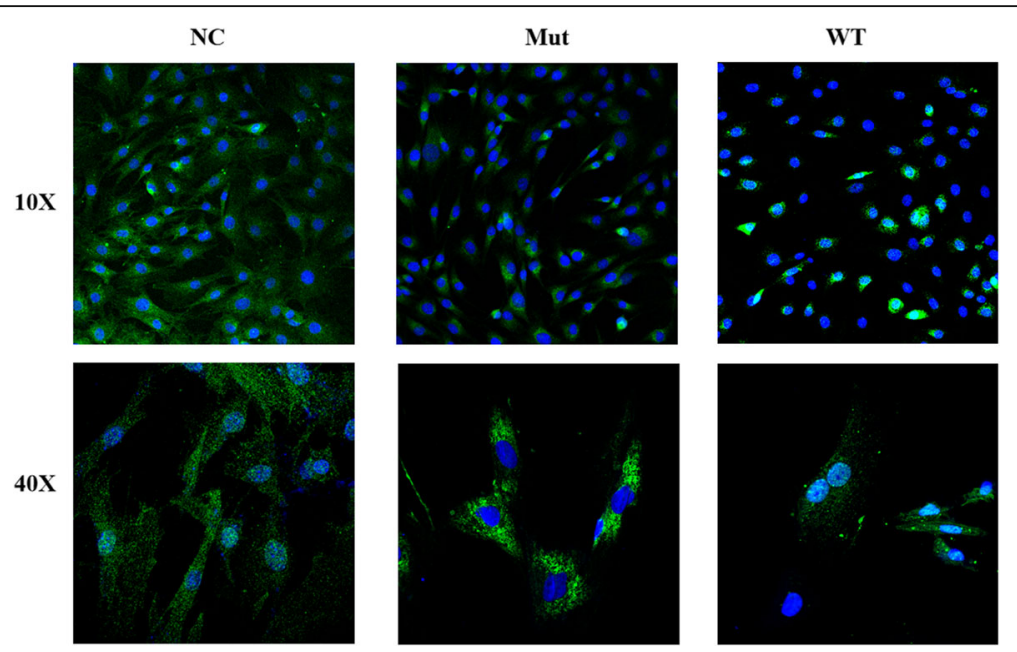

Fig. 3 Subcellular localization of the RUNX2 mutant protein (NC, Mut, WT indicate control GFP, mutant Gly223Val RUNX2 and wild-type RUNX2, respectively. Magnification 10X and 40X)

The effect of the RUNX2 variants on protein structure Among these variants, there were three variants changing the number of $\mathrm{H}$-bonds in RUNX2 protein, including two variants increasing $\mathrm{H}$-bonds (c.574G > A, p.Gly192Arg; c.668G > T, p.Gly223Val) and one variant decreasing $\mathrm{H}-$ bonds (c.673 $\mathrm{C}>\mathrm{T}$, p.Arg225Trp). In addition, there were four variants (c.722_725delTGTT, p.Leu241Serfs*8; c.231_ 232delTG, Ala78Glyfs*82; c.577C > T, p.Arg193*; c.909C > G, p.Tyr303*) decreasing the number of amino acids in RUNX2 protein.

\section{Subcellular localization of the RUNX2 mutant protein}

To further explore the function of the missense mutation (c.668G > T, p.Gly223Val) not reported, the widetype and mutant RUNX2 proteins binding green fluorescent protein (GFP) were constructed and transiently transfected into human osteosarcoma U2OS. The result showed that the Gly223Val mutation could affect the subcellular distribution of RUNX2 protein and made RUNX2 protein unable to quantitatively accumulate in the nucleus (Fig. 3).

\section{Discussion}

CCD is a skeletal dysplasia that represents a continuum of clinical findings ranging from classical CCD (dental abnormalities, hypoplastic or aplastic clavicles, and delayed closure of the cranial sutures) to mild CCD to isolated dental anomalies without other skeletal features. To date, no formal clinical diagnostic criteria for CCD have been established. Due to CCD inherited in an autosomal dominant manner, each child of an individual with CCD has a 50\% chance of inheriting the pathogenic variant. If the pathogenic variant in the family is known, prenatal diagnosis for pregnancies at increased risk will be possible. Many kinds of molecular testing approaches, including single-gene testing, karyotype analysis and a multigene panel, can be currently used to detect the variants leading to CCD. For single-gene testing, sequence analysis of RUNX2 gene is performed first and followed by gene-targeted deletion/duplication analysis if no pathogenic variant is identified. For karyotype analysis, if RUNX2 testing is not diagnostic and strong suspicion persists in an individual with CCD features who also has multiple congenital anomalies and/or developmental delay, a karyotype analysis may be considered to evaluate complex chromosome rearrangements or translocations that involve RUNX2 locus but do not result in RUNX2 copy number changes $[25,26]$. In addition, a multigene panel that includes RUNX2 and other genes of interest may also be considered.

In the present study, we utilized targeted highthroughput DNA sequencing or Sanger sequencing (single-gene testing) techniques to analyze genetic variants in seven CDD children, and found seven different variants

Table 4 Summarization of RUNX2 gene variants in the HGMD and current study

\begin{tabular}{llll}
\hline Variant type & \multicolumn{3}{l}{ Number of variants (\%) } \\
\cline { 2 - 4 } & HGMD & The current study & Total \\
\hline Missense/nonsense & $77(41.8 \%)$ & $5(71.4 \%)$ & $82(42.9 \%)$ \\
Splicing & $11(6.0 \%)$ & - & $11(5.8 \%)$ \\
Small deletions & $44(23.9 \%)$ & $2(28.6 \%)$ & $46(24.1 \%)$ \\
Small insertions & $22(12.0 \%)$ & - & $22(11.5 \%)$ \\
Small indels & $2(1.1 \%)$ & - & $2(1.0 \%)$ \\
Gross deletions & $17(9.2 \%)$ & - & $17(8.9 \%)$ \\
Gross insertions/duplications & $5(2.7 \%)$ & - & $5(2.6 \%)$ \\
Complex rearrangements & $4(2.2 \%)$ & - & $4(2.1 \%)$ \\
Repeat variations & $2(1.1 \%)$ & - & $2(1.0 \%)$ \\
\hline
\end{tabular}




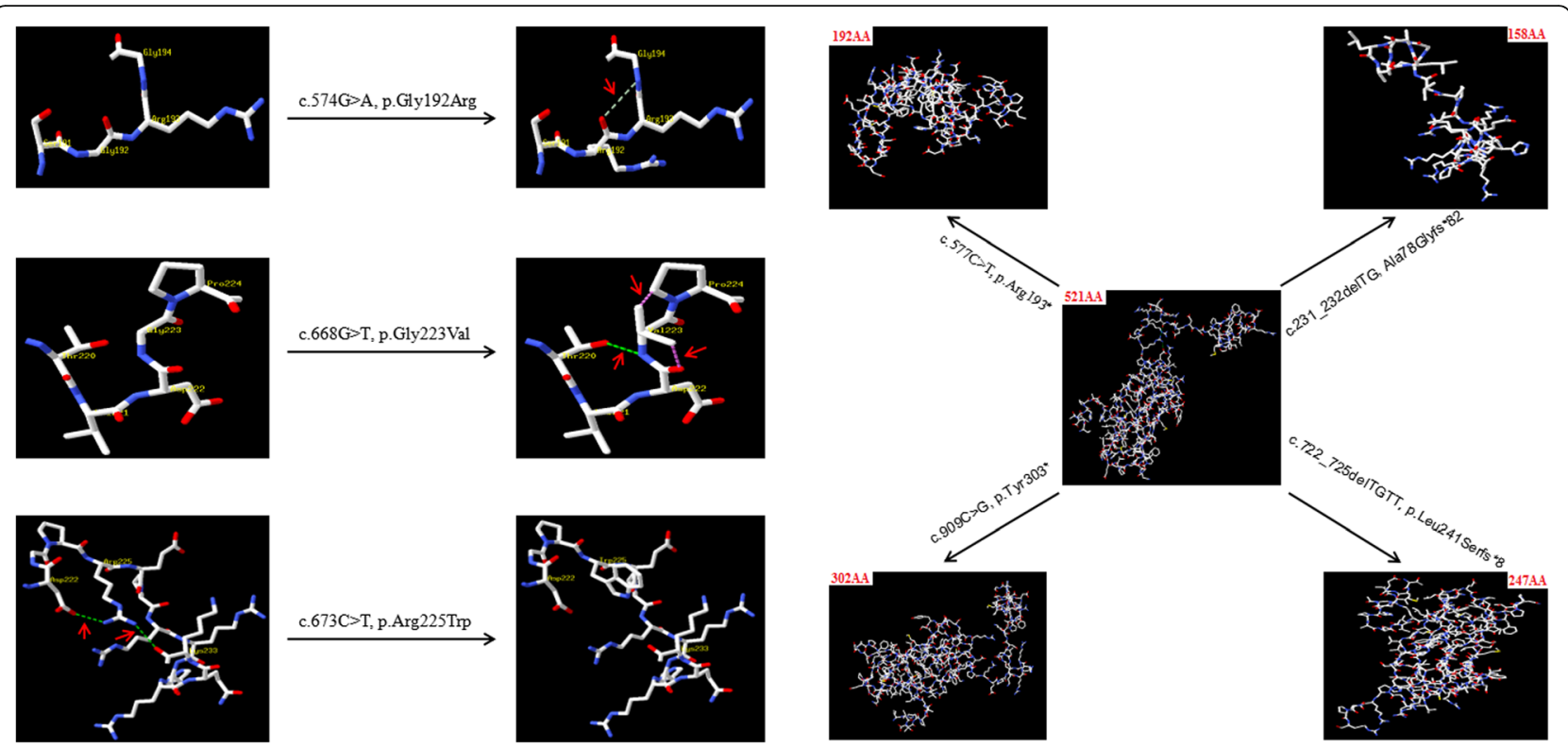

Fig. 4 The effect of RUNX2 gene variants on protein structure (The red arrow indicates the H-bonds)

in RUNX2 gene, including four novel variants (c.722 725delTGTT, p.Leu241Serfs*8; c.231_232delTG, Ala78Glyfs*82; c.909C > G, p.Tyr303*; c.668G > T, p.Gly223$\mathrm{Val}$ ) and three reported variants (c.577C > T, p.Arg193\%; c.574G > A, p.Gly192Arg; c.673 C > T, p.Arg225Trp) [27-29], which were all located in the transactivation region (Fig. 2). The bioinformatics analysis indicated that these variants were disease-causing, damaging and/or probably damaging variants. According to ACMG, six variants (c.574G > A, p.Gly192Arg; c.673 C > T, p.Arg225Trp; c.577C > T, p.Arg193*; c.722_725delTGTT, p.Leu241Serfs"8; c.231_232delTG, Ala78Glyfs*82; c.909C > G, p.Tyr303*) were classified as pathogenic variants, and one variant (c.668G > T, p.Gly223Val) as likely pathogenic variant. In addition, all variants were de novo except the following two variants: c.909C > G, p.Tyr303* and c.668G > T, p.Gly223Val. Thereinto the former variant (c.909C $>$ G, p.Tyr303*) was inherited from the patient's father, who is also a CCD patient carried a de novo heterozygous RUNX2 variant. The clinical features of the father included short stature and CCD, which were very similar to those of his 3-year-old son. The latter variant (c.668G > T, p.Gly223Val) was inherited from the patient's mother with CCD, who carried a maternal inherited and heterozygous RUNX2 variant. Both of them also showed similar clinical phenotypes, such as short stature and CCD. By summarizing RUNX2 variants in HGMD and the current study, we found nine variant types, such as missense/nonsense, splicing, small deletions/insertions, gross insertions/duplications. Thereinto missense/nonsense variant was the most common variant type of RUNX2 gene (Table 4). A single amino acid (Gly) substitution at position 332 in RUNX2 protein was found not only in our lab (c.668G $>$ T, p.Gly223Val), but also in Ott' s study (c.667G > A, p.Gly223Arg) [1]. In addition, protein structure prediction showed that these variants could change the number of H-bonds or amino acids in RUNX2 protein (Fig. 4), suggesting that these variants played an important role in regulating the effective structure and function of RUNX2 protein. The experimental result showed that Gly223Val mutation, located in nuclear localization sequence (NLS) [29, 30], could affect the subcellular distribution of RUNX2 protein. The mutation made RUNX2 protein unable to quantitatively accumulate in the nucleus.

In conclusion, the present study reveals some novel genetic causes of CDD, which not only expands the pathogenic variant spectrum of $R U N X 2$ gene but also will contribute to the diagnosis of CCD and better genetic counseling in the future.

\section{Abbreviations}

ACMG: American College of Medical Genetics and Genomics; CCD: Cleidocranial dysplasia; EVS: Exome Variant Server; ExAC: Exome Aggregation Consortium; RUNX2: Runt-related transcription factor 2

\section{Acknowledgments}

We thank the patients and their families for participating in our study.

\section{Authors' contributions}

$\mathrm{XG}$ and $\mathrm{KL}$ analyzed and interpreted the patients' data and were major contributors in writing the manuscript. All authors read and approved the final manuscript.

\section{Funding}

This work is supported by grants from the "Youth Research Project of the Shanghai Municipal Health and Family Planning Commission" (No. 
20184Y0348, to GXR); the "National Natural Science Foundation of China" (No.81800780 to GXR; No.81670812 and No.81873671, to YYG); the "Jiaotong University Cross Biomedical Engineering" (No.YG2017MS72, to YYG); the "Shanghai Municipal Commission of Health and Family Planning" (No.201740192, to YYG); the "Shanghai Shen Kang Hospital Development Center new frontier technology joint project" (No.SHDC12017109, to YYG).

\section{Availability of data and materials}

The datasets used and/or analysed during the current study are available from the corresponding author on reasonable request.

\section{Ethics approval and consent to participate}

This study was carried out in accordance with the Code of Ethics of the World Medical Association (Declaration of Helsinki) and was certified by the Ethics Committee of Xinhua Hospital affiliated with the Shanghai Jiaotong University School of Medicine. Written informed consent was obtained from the patient's parents.

\section{Consent for publication}

Not applicable.

\section{Competing interests}

The authors declare that they have no competing interests.

\section{Author details}

'Department of Pediatric Endocrinology and Genetics, Shanghai Institute for Pediatric Research, Xinhua Hospital, School of Medicine, Shanghai Jiaotong University, Shanghai 200092, China. ${ }^{2}$ The Affiliated Yantai Yuhuangding Hospital of Qingdao University, Qingdao, Shandong, China.

\section{Received: 4 March 2019 Accepted: 9 September 2019}

\section{Published online: 16 September 2019}

\section{References}

1. Ott CE, Leschik G, Trotier F, Brueton L, Brunner HG, Brussel W, et al. Deletions of the RUNX2 gene are present in about 10\% of individuals with cleidocranial dysplasia. Hum Mutat. 2010;31(8):E1587-93.

2. Otto $\mathrm{F}$, Kanegane $\mathrm{H}$, Mundlos $\mathrm{S}$. Mutations in the RUNX2 gene in patients with cleidocranial dysplasia. Hum Mutat. 2002;19(3):209-16.

3. Otto F, Thornell AP, Crompton T, Denzel A, Gilmour KC, Rosewell IR, et al. Cbfa1, a candidate gene for cleidocranial dysplasia syndrome, is essential for osteoblast differentiation and bone development. Cell. 1997;89(5):765-71.

4. Levanon D, Negreanu V, Bernstein Y, Bar-Am I, Avivi L, Groner Y. AML1, AML2, and AML3, the human members of the runt domain gene-family: cDNA structure, expression, and chromosomal localization. Genomics. 1994; 23(2):425-32.

5. Yoshida T, Kanegane $H$, Osato M, Yanagida M, Miyawaki $T$, Ito $Y$, et al. Functional analysis of RUNX2 mutations in Japanese patients with cleidocranial dysplasia demonstrates novel genotype-phenotype correlations. Am J Hum Genet. 2002;71(4):724-38.

6. Ogawa E, Maruyama M, Kagoshima H, Inuzuka M, Lu J, Satake M, et al. PEBP2/PEA2 represents a family of transcription factors homologous to the products of the Drosophila runt gene and the human AML1 gene. Proc Natl Acad Sci U S A. 1993:90(14):6859-63.

7. Kagoshima H, Shigesada K, Satake M, Ito Y, Miyoshi H, Ohki M, et al. The runt domain identifies a new family of heteromeric transcriptional regulators. Trends Genet. 1993;9(10):338-41.

8. Ducy P, Zhang R, Geoffroy V, Ridall AL, Karsenty G. Osf2/Cbfa1: a transcriptional activator of osteoblast differentiation. Cell. 1997;89(5):747-54.

9. Thirunavukkarasu K, Mahajan M, McLarren KW, Stifani S, Karsenty G. Two domains unique to osteoblast-specific transcription factor Osf2/Cbfa1 contribute to its transactivation function and its inability to heterodimerize with Cbfbeta. Mol Cell Biol. 1998;18(7):4197-208.

10. Pande S, Browne G, Padmanabhan S, Zaidi SK, Lian JB, van Wijnen AJ, et al. Oncogenic cooperation between PI3K/Akt signaling and transcription factor Runx2 promotes the invasive properties of metastatic breast cancer cells. J Cell Physiol. 2013:228(8):1784-92.

11. Levanon D, Goldstein RE, Bernstein Y, Tang H, Goldenberg D, Stifani S, et al. Transcriptional repression by AML1 and LEF-1 is mediated by the TLE/ Groucho corepressors. Proc Natl Acad Sci U S A. 1998;95(20):11590-5.
12. Komori T, Yagi H, Nomura S, Yamaguchi A, Sasaki K, Deguchi K, et al. Targeted disruption of Cbfa1 results in a complete lack of bone formation owing to maturational arrest of osteoblasts. Cell. 1997;89(5):755-64.

13. Komori T. Runx2, an inducer of osteoblast and chondrocyte differentiation. Histochem Cell Biol. 2018;149(4):313-23.

14. Peng YJ, Chen QY, Fu DJ, Liu ZM, Mao TT, Li J, et al. A novel gene mutation of Runx2 in cleidocranial dysplasia. J Huazhong Univ Sci Technolog Med Sci. 2017;37(5):772-6.

15. Zhang $T$, Wu J, Zhao $X$, Hou F, Ma T, Wang $H$, et al. Whole-exome sequencing identification of a novel splicing mutation of RUNX2 in a Chinese family with cleidocranial dysplasia. Arch Oral Biol. 2019;100:49-56.

16. Çamtosun E, Akıncı A, Demiral E, Tekedereli İ, Sığırcı A. A cleidocranial dysplasia case with a novel mutation and growth velocity gain with growth hormone treatment. J Clin Res Pediatr Endocrinol. 2018;11(3):301-5. [Epub ahead of print].

17. Ma D, Wang X, Guo J, Zhang J, Cai T. Identification of a novel mutation of RUNX2 in a family with supernumerary teeth and craniofacial dysplasia by whole-exome sequencing: a case report and literature review. Medicine (Baltimore). 2018;97(32):e11328.

18. Hashmi JA, Almatrafi A, Latif M, Nasir A, Basit S. An 18 bps in-frame deletion mutation in RUNX2 gene is a population polymorphism rather than a pathogenic variant. Eur J Med Genet. 2019:62(2):124-8.

19. Zeng L, Wei J, Zhao N, Sun S, Wang Y, Feng H. A novel 18-bp in-frame deletion mutation in RUNX2 causes cleidocranial dysplasia. Arch Oral Biol. 2018;96:243-8

20. Schwarz JM, Cooper DN, Schuelke M, Seelow D. MutationTaster2: mutation prediction for the deep-sequencing age. Nat Methods. 2014;11(4):361-2.

21. Choi Y, Chan AP. PROVEAN web server: a tool to predict the functional effect of amino acid substitutions and indels. Bioinformatics. 2015;31(16): 2745-7.

22. Adzhubei IA, Schmidt S, Peshkin L, Ramensky VE, Gerasimova A, Bork P, et al. A method and server for predicting damaging missense mutations. Nat Methods. 2010:7(4):248-9.

23. Waterhouse A, Bertoni M, Bienert S, Studer G, Tauriello G, Gumienny R, et al SWISS-MODEL: homology modelling of protein structures and complexes. Nucleic Acids Res. 2018;46(W1):W296-303.

24. Guex N, Peitsch MC. SWISS-MODEL and the Swiss-PdbViewer: an environment for comparative protein modeling. Electrophoresis. 1997; 18(15):2714-23.

25. Purandare SM, Mendoza-Londono R, Yatsenko SA, Napierala D, Scott DA, Sibai $T$, et al. De novo three-way chromosome translocation $46, X Y, t(4 ; 6 ;$ 21)(p16;p21.1; q21) in a male with cleidocranial dysplasia. Am J Med Genet A. $2008 ; 146 \mathrm{~A}(4): 453-8$

26. Northup JK, Matalon R, Lockhart LH, Hawkins JC, Velagaleti GV. A complex chromosome rearrangement, der (6) ins (6)(p21.1q25.3q27)inv (6)(p25.3q27), in a child with cleidocranial dysplasia. Eur J Med Genet. 2011:54(4):e394-8.

27. Zhou G, Chen Y, Zhou L, Thirunavukkarasu K, Hecht J, Chitayat D, et al. CBFA1 mutation analysis and functional correlation with phenotypic variability in cleidocranial dysplasia. Hum Mol Genet. 1999;8(12):2311-6.

28. Puppin C, Pellizzari L, Fabbro D, Fogolari F, Tell G, Tessa A, et al. Functional analysis of a novel RUNX2 missense mutation found in a family with cleidocranial dysplasia. J Hum Genet. 2005;50(12):679-83.

29. Quack I, Vonderstrass B, Stock M, Aylsworth AS, Becker A, Brueton L, et al. Mutation analysis of core binding factor $A 1$ in patients with cleidocranial dysplasia. Am J Hum Genet. 1999;65(5):1268-78.

30. Kanno T, Kanno Y, Chen LF, Ogawa E, Kim WY, Ito Y. Intrinsic transcriptional activation-inhibition domains of the polyomavirus enhancer binding protein 2/core binding factor alpha subunit revealed in the presence of the beta subunit. Mol Cell Biol. 1998;18(5):2444-54.

\section{Publisher's Note}

Springer Nature remains neutral with regard to jurisdictional claims in published maps and institutional affiliations. 